\title{
Sheath voltage ratio for asymmetric rf discharges
}

\author{
M. V. Alves, ${ }^{\text {a) }}$ M. A. Lieberman, V. Vahedi, and C. K. Birdsall \\ Department of Electrical Engineering and Computer Sciences and the Electronics Research Laboratory, \\ University of California, Berkeley, California 94720
}

(Received 22 June 1990; accepted for publication 3 January 1991)

\begin{abstract}
Spherical and cylindrical many-particle models are used to simulate rf (radio frequency) discharges in which the rf powered and the grounded electrodes have different areas. This asymmetry determines the magnitude of the average plasma-to-electrode voltage $V_{a}$ (the ion bombarding energy) at the smaller powered electrode, which is a critical process parameter. A collisionless uniform ionization discharge model predicts that the voltage ratio $V_{a} / V_{b}$ scales as the fourth power of the electrode area ratio $A_{b} / A_{a}$, where $V_{b}$ is the potential drop at the other electrode. However, measurements indicate a much weaker dependence of $V_{a} / V_{b}$ on the area ratio, which is also observed in our simulations. Over a limited range of area ratios it was found that the power dependence was close to one, in agreement with a local ionization discharge model. The simulation codes used are PDC1 (plasma device cylindrical one-dimensional) and PDS1 (plasma device spherical one-dimensional).
\end{abstract}

\section{INTRODUCTION}

Capacitive discharges are widely used for materials processing in the electronics industry. The discharges are generally asymmetric: the radio-frequency (rf) powered electrode and the grounded electrode have different area, $A_{a}$ and $A_{b}$, respectively, with $A_{a}$ typically less than $A_{b}$. This asymmetry determines the magnitude of the plasma-toelectrode voltage $V_{a}$ (the ion bombarding energy) at the powered electrode, which is a critical parameter for VLSI (very large scale integrated) processing. A simple collisionless discharge model ${ }^{1}$ predicts the scaling $V_{a} / V_{b}$ $=\left(A_{b} / A_{a}\right)^{4}$, where $V_{b}$ is the potential drop at the other electrode. However, measurements indicate a much weaker dependence of $V_{a} / V_{b}$ on the area ratio. ${ }^{2}$ For capacitive discharges, almost all the voltage drop appears across thin sheaths, having thicknesses $s_{a}$ and $s_{b}$, at the powered and grounded electrodes. In most cases there is an external blocking capacitor, $C_{B}$, having negligible impedance at the rf driving frequency, in series with the driven electrode. Between the sheaths there is a thermal plasma or "glow" region with a thickness $d \geqslant s$ which serves to maintain the discharge by means of ion generation there, balancing the loss of particles to the electrodes. These processes of ionization and loss determine the plasma density profile and, in particular, the densities $n_{s a}$ and $n_{s b}$ at the two sheath edges near the powered and grounded electrodes, which are found not to be equal as is often assumed. In turn, these densities determine the sheath thickness and the sheath voltages $V_{a}$ and $V_{b}$.

In this work, we present computational results obtained using spherical and cylindrical many-particle simulation models which we compare with theoretical results. The simulation codes ${ }^{3}$ are PDC1 (plasma device cylindrical one-dimensional) and PDS1 (plasma device spherical one-dimensional) which utilize particle-in-cell techniques plus Monte Carlo simulation of electron-neutral (elastic, excitation, and ionization) and ion-neutral (scattering and charge-exchange) collisions. ${ }^{4}$

\section{SIMULATION PROCEDURE}

PDC1 and PDS1 are one-dimensional, radial, electrostatic codes simulating a plasma contained between concentric spheres or infinite cylinders that can be coupled to an external RLC circuit and/or an rf source, ${ }^{4}$ as shown in Fig. 1. The codes use particle-in-cell techniques ${ }^{5}$ and were developed from the simulation code PDW1 (plasma device workshop one-dimensional) ${ }^{6}$

Particles of finite thickness (cylindrical shells in PDC1 and spherical shells in PDS1) are placed in a gridded system, and mathematical artifice, and weighted to the grid to obtain the density $\rho_{j}$ at the grid points. The assumption that the particles have uniform density allows us to use the area of rings in the cylindrical model, and the volume of shells in the spherical one, to weight the charges to the grids as shown in Fig. 2.

The density on the grid is used to solve Poisson's equation, $\nabla^{2} \Phi=-\rho / \epsilon$, which in a finite difference form is given by

$$
\begin{gathered}
\left(r_{j}-0.5\right) \Phi_{j-1}-2 r_{j} \Phi_{j}+\left(r_{j}+0.5\right) \Phi_{j+1}=\frac{-r_{j} \Delta r^{2} \rho_{j}}{\epsilon_{0}}, \\
j>0
\end{gathered}
$$

and

$$
\Phi_{0}-\Phi_{1}=\Delta r \frac{\left(r_{0}+0.25\right) \Delta r p_{0} / 2+r_{0} \sigma_{a}}{\left(r_{0}+0.5\right) \epsilon_{0}}
$$

in the cylindrical model and

'Permanent addres: INPE, P.O. Box 515, S. J. dos Campos, San Paulo, 12201, Brazil. 


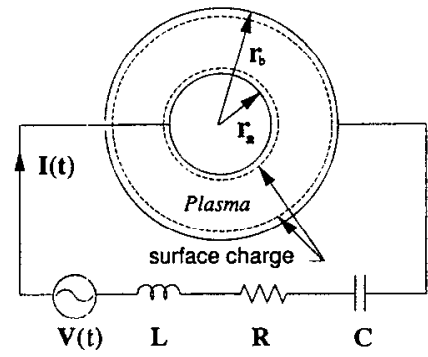

FIG. 1. Cylindrical (spherical) plasma device with an external RLC circuit.

$$
\begin{gathered}
r_{j-1 / 2}^{2} \Phi_{j-1}-\left(r_{j+1 / 2}^{2}+r_{j-1 / 2}^{2}\right) \Phi_{j}+r_{j+1 / 2}^{2} \Phi_{j+1} \\
==-\left(r_{j+1 / 2}^{3}-r_{j-1 / 2}^{3}\right) \frac{\Delta r^{2} \rho_{j}}{3 \epsilon_{0}}, \quad j>0,
\end{gathered}
$$

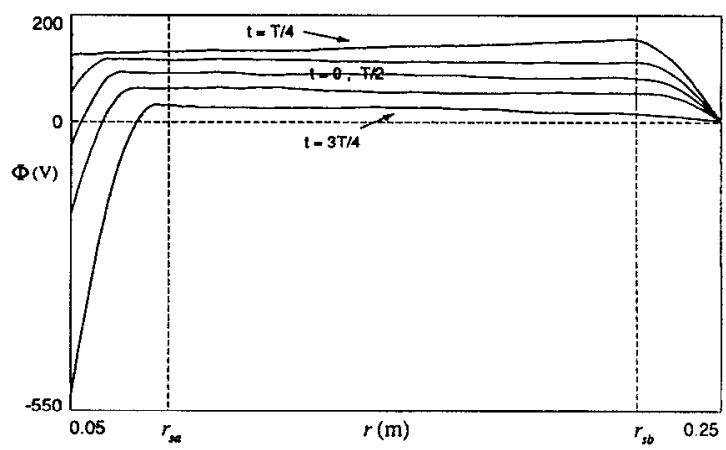

and

$$
\Phi_{0}-\Phi_{1}=\Delta r \frac{\left(r_{1 / 2}^{3}-r_{0}^{3}\right) \Delta r \rho_{0} / 3+r_{0}^{2} \sigma_{a}}{r_{1 / 2}^{2} \epsilon_{0}}, j=0,
$$

for the spherical model. In the previous equations, $\Delta r$ is the grid spacing, and $r_{j}$ denotes grid point position normalized by $\Delta r$. We will also assume $\Phi_{\mathrm{NC}}=0$ as both a boundary condition and a definition for a reference potential. The other boundary condition is given by the equation involving $\Phi_{0}$ obtained from applying Gauss' law at the cylindrical or spherical surface at $j=1 / 2,{ }^{5}$ including the surface charge densities on the electrode.

Poisson's equation can be combined with the circuit equation and expressed in the matrix form. ${ }^{7} \mathrm{PDCl}$ and PDS I solve this matrix equation every time step to obtain the spatial potential and the current in the external circuit self-consistently to second order accuracy. The matrix equation can be solved using any algorithm optimized for tridiagonal matrices. ${ }^{8}$

Once the electric potential as the grid is known, the electric field on the grid can be obtained from $E=-\nabla \Phi$. The particles are moved by obtaining $E$ at each particle position using the same used to weight particles and applying this to the Newton-Lorentz force equation:

$$
\frac{d \mathbf{v}_{i}}{d t}=\frac{q_{i}}{m_{i}}\left[\mathbf{E}\left(\mathbf{r}_{i}\right)+\mathbf{v}_{i} \times \mathbf{B}\right]
$$

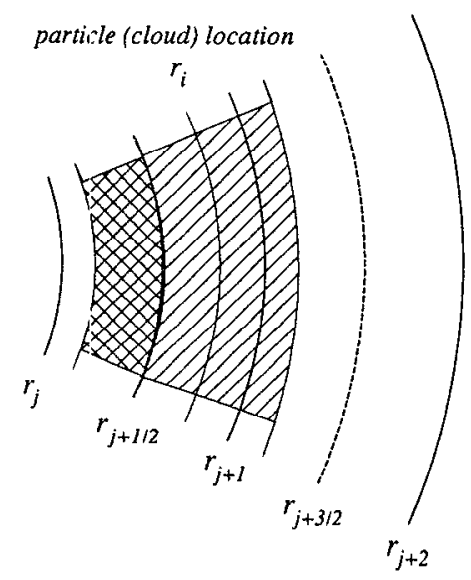

FIG. 2. Particle weighting to $\mathbf{r}_{j}$ grid points, using area (for cylindrical) or volume (for spherical) ratio. The particle at $\mathbf{r}_{i}$ extends over two cells $(j$ and $j+1$ ) with the crosshatched fraction assigned to $j$ and the slanted fraction assigned to $j+1$.
FIG. 3. Typical potential during an if period for an asymmetric discharge. This particular simulation result was obtained from PDC1 with argon as the background neutral gas at $p=30 \mathrm{mTorr}$ and $A_{b} / A_{a}=5$.

In order to model weakly ionized discharges with large neutral populations, accurate models of elastic, excitation, and ionizing electron-neutral collisions and scattering, and charge-exchange ion-neutral collisions are included. ${ }^{4}$

\section{THEORETICAL MODELS}

In recent years, some analytic models have been developed for the sheath voltage ratio $V_{a} / V_{b}$. A model based on probe theory, which includes the effect of the floating potential and fits some of the experiments, was developed by Pointu, ${ }^{9}$ who assumes collisionless sheaths and equal ion current densities at the electrode surfaces. A onedimensional spherical shell model ${ }^{10}$ and a finite length cylindrical model ${ }^{11}$ have also been developed. These models incorporate various assumptions for the sheath and glow physics, yielding scalings more in agreement with measurements. We compare our simulation results for the cylindrical and the spherical cases with modified versions of these models.

The relation between the if voltage amplitude $\widetilde{V}_{a}$ and the dc voltage drop $V_{a}$ is complicated. ${ }^{12}$ If the voltage across a sheath is of the form $V=V_{\mathrm{dc}}=V_{0} \sin (\omega t)$, and if the Maxwell-Boltzmann distribution holds for the electrons $^{13}$ we can write: ${ }^{14}$

$$
V_{a}=T_{e} \ln \left[I_{0}\left(\widetilde{V}_{a} / T_{e}\right)\right]+\frac{T_{e}}{2} \ln (M / 2 \pi m),
$$

where $T_{e}$ is the electron temperature (in units of volts) and $M / m$ is the ion-to-electron mass ratio. For $\widetilde{V}_{a}>T_{e}$, we can expand the $I_{0}$ modified Bessel function to obtain:

$$
\widetilde{V}_{a}=V_{a}-V_{f a},
$$

where $V_{f a}$ is called the floating potential, because it includes the floating potential in the absence of any rf voltage $^{13}$ given by

$$
V_{f a}=\frac{T_{e}}{2} \ln \left(\frac{M T_{e}}{4 \pi^{2} m \tilde{V}_{a}}\right) .
$$

Figure 3 shows a typical plot of potential versus position during an rf period and some definitions used in this 
(a)

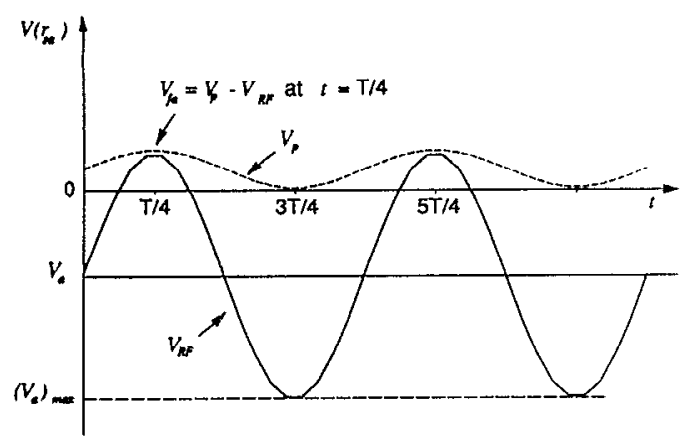

(b)

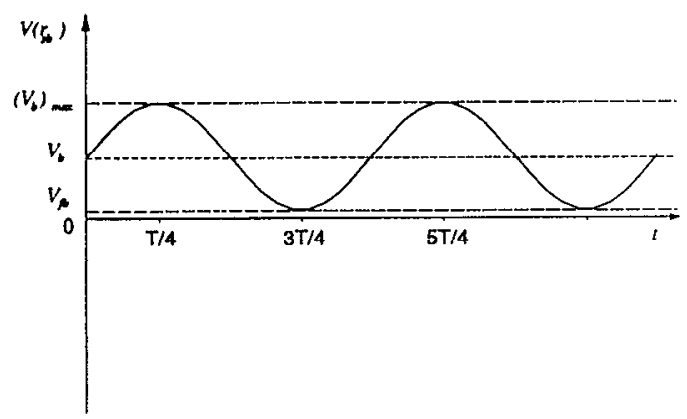

FIG. 4. (a) Time variation of the potential at the powered electrode; (b) time variation of the potential at the sheath-edge position close to the ground electrode.

work. From Fig. 3 we can construct Figs. 4(a) -4(b) and obtain the dc components across the sheaths. Since the glow region cannot sustain a potential larger then a few multiples of $T_{e}$, because of its large conductivity, the position of the sheath-edge $\left(r_{s a}\right.$ and $\left.r_{s b}\right)$ is then assumed to be the position where the potential becomes fairly constant. There is a capacitive coupling between the rf generator and the driven electrode, so no net current can flow through the drive electrode circuit and the plasma potential must approach the potential of the driven electrode for a brief period during each if cycle to allow electrons to reach the driven electrode. Similarly, no net current can flow to ground, and the plasma potential also approaches ground potential for a brief period during each cycle. These variations are shown in Figs. 4(a)-4(b). The difference between the plasma potential and the driven electrode potential at $T / 4$ is assumed to be $V_{f a}$; the plasma potential at time $3 T / 4$ is assumed to be $V_{f b}$ for modeling purposes.

For high voltage capacitive sheaths, $V_{f a}<V_{a}$ and $\widetilde{V}_{a}$ $\approx V_{a}$. Within these limits, assuming $C \propto A / s$ and knowing that $C=Q / C$ the of current density is related to the dc sheath voltage and sheath thickness by a capacitive scaling

$$
J_{a}(\bar{x}) \propto \frac{V_{a}}{s_{a}(\bar{x})},
$$

where $s(\bar{x})$ is the sheath thickness and $\bar{x}$ specifies the position on the electrode surface and the voltages are independent of $\bar{x} \cdot{ }^{12}$ The total if current $I_{a}$ flowing to the powered electrode is
TABLE I. Exponents for various sheath scaling laws and for the zeroorder relationship between the sheath voltage ratio and area ratio.

\begin{tabular}{lccc}
\hline \hline Sheath ion model & $p$ & $q$ & $\frac{q}{1+p+q}$ \\
\hline Collisionless & $1 / 2$ & 4 & $8 / 11(0.7273)$ \\
Elastic scattering & $1 / 3$ & 3 & $9 / 13(0.6923)$ \\
Resonant charge exchange & $2 / 5$ & $5 / 2$ & $25 / 39(0.641)$ \\
\hline \hline
\end{tabular}

$$
I_{a} \propto \int_{A_{a}} J_{a}(\bar{x}) d^{2} x .
$$

The separation into distinct sheath and glow regions is somewhat arbitrary. We use the Bohm sheath criterion ${ }^{15}$ to define the plasma-sheath edge, which means the ion velocity, $u$, is equal to the Bohm velocity $u=u_{B}$ $=\left(e T_{e} / M\right)^{1 / 2}$, where $e / M$ is the ion charge-to-mass ratio.

The relationship between the density $n_{s}(\bar{x})$ at the plasma-sheath edge, the dc glow-to-electrode voltage, $V_{a}$, and the sheath thickness, $s(\bar{x})$, depends on the assumptions we make for the sheath. For an ion collisionless sheath, we assume a Child's law dependence ${ }^{10}$

$$
n_{s a}(\bar{x}) \propto \frac{V_{a}^{3 / 2}}{s_{a}^{2}(\bar{x})} .
$$

For an ion collisional resonant charge transfer sheath, we assume that ${ }^{10}$

$$
n_{s a}(\bar{x}) \propto \frac{V_{a}^{3 / 2}}{s_{a}^{5 / 2}(\bar{x})} .
$$

For an ion collisional elastic scattering sheath we assume that $^{16}$

$$
n_{s a}(\bar{x}) \propto \frac{V_{a}^{2}}{s_{a}^{3}(\bar{x})} .
$$

Expressions similar to (5)-(7) are obtained for the grounded electrode and specified by subscript $b$. Equating $I_{a}$ to $I_{b}$ by conservation of current, we then obtain the fundamental scaling formula for high voltage sheaths,

$$
\left(\frac{V_{a}}{V_{b}}\right)_{0}=\left(\frac{\int_{A_{b}} n_{s b}^{p}(\bar{x}) d^{2} x}{\int_{A_{a}} n_{s a}^{p}(\bar{x}) d^{2} x}\right)^{q},
$$

where $p$ and $q$ depend on the sheath model, and are given in Table I.

For large area ratios, the voltage across the large area electrode is low and the floating potential in (2) cannot be neglected. In that case $\widetilde{V}_{a}$ must be used in Eqs. (5)-(7). Following the preceding analysis we obtai:

$$
\frac{V_{a}}{V_{b}}=\left(\frac{1-V_{f b} / V_{b}}{1-V_{f a} / V_{a}}\right)^{q}\left(\frac{V_{a}}{V_{b}}\right)_{0}
$$

where now $V_{a}$ and $V_{b}$ are related to the peak-to-peak voltage $\widetilde{V}_{p p}$, appearing at the target electrode, by

$$
V_{a}+V_{b}=V_{f a}+V_{f b}+\widetilde{V}_{p p} / 2 .
$$



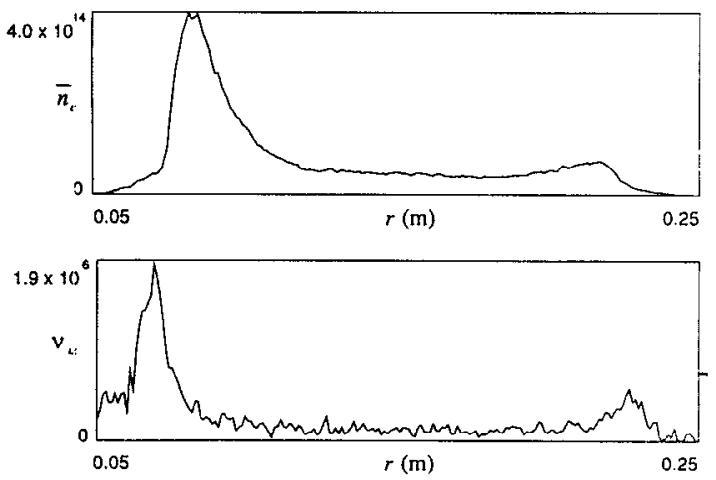

FIG. 5. (a) Typical spatial variation of the charge electron density averaged over an $\mathrm{rf}$ period and (b) the ionization rate, $v_{i r}$ in the cylindrical system (cumulative in time). These results were obtained from PDC1 with argon (collisional ions) as the background neutral gas at $p=30$ mTorr and $A_{b} / A_{a}=5$.

For most simulation parameters, our results indicate that the ionization rate is highest near the sheaths; e.g., in the cylindrical case shown in Fig. 5(b). This local ionization is due to stochastic electron heating produced by the oscillating sheaths and occurs when the mean free path for ionization by electrons is smaller than the discharge length. Figure 5(a) shows the electron number density across the system, averaged over an if period, $\bar{n}_{e}$. The number of ionizations occurring in a grid cell per time interval is given by $\bar{n}_{e} v_{i z} V_{j}$ where $V_{j}$ is the cell volume and $v_{i z}$ is the ionization rate shown in Fig. 5(b); this rate is obtained cumulatively, over the entire run.

Assuming that local ionization near each sheath dominates the overall ionization, we obtain the scaling ${ }^{10}$

$$
\frac{n_{s a}}{n_{s b}}=\frac{V_{a}}{V_{b}} .
$$

Using (11) in (8) we obtain:

$$
\left(\frac{V_{a}}{V_{b}}\right)_{0}=\left(\frac{A_{b}}{A_{a}}\right)^{q / 1+p+q} \text {. }
$$

Table I shows the values for $p, q$, and the exponent in Eq. (12), $q /(1+p+q)$ for the various sheath models.

Solving (9), (10), and (12) numerically, we obtain the values for $V_{a} / V_{b}$ in terms of $A_{b} / A_{a}$. Observe that there is a dependence of $V_{a} / V_{b}$ on the electron temperature, $T_{e}$, due to the dependence of the floating potential on $T_{e}$.

\section{SIMULATION RESULTS FOR VOLTAGE RATIOS}

Several runs were made with PDC1 and PDS1 for different area ratios with two kinds of background gas (hydrogen and argon) at $p=30$ mTorr, with and without ion-neutral collisions. The area ratio, defined as $A_{b} / A_{a}$, was varied from 1.3 to 15 . The inner and outer radii were chosen to maintain the same plasma length $l$ where $l=r_{b}$ $-r_{a}(20 \mathrm{~cm})$ for all cases. The external capacitance was chosen so that the blocking capacitor had negligible im-

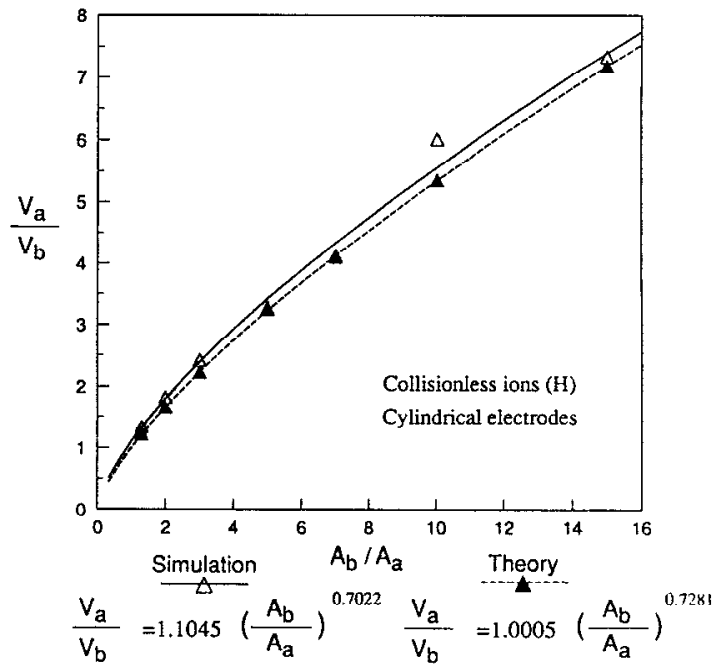

FIG. 6. $V_{a} / V_{b}$ vs $A_{b} / A_{a}$ for hydrogen gas at $30 \mathrm{mT}$ Torr (collisionless ions in the simulation and the theory assumes a collisionless sheath model). The equations are the best fits to the data.

pedance at the rf driving frequency compared to the vacuum capacitance of the discharge.

In the simulation, the potential at the outer electrode is always zero $\left(\Phi_{\mathrm{NC}}=0\right.$, reference potential $)$. In the figures, "collisionless ions" and "ion-neutral collisions" mean, respectively, that ion-neutral collisions in the simulations are either turned off or on, across the system. Electron-neutral scattering, excitation and ionization collisions are always present. The plasma characteristics will determine the collisionality regime in the sheath.

Figures 6 and 7 show simulation and analytic results for cylindrical electrodes plotting $V_{a} / V_{b}$ vs $A_{b} / A_{a}$ in hydrogen and argon. The analytic points were obtained by

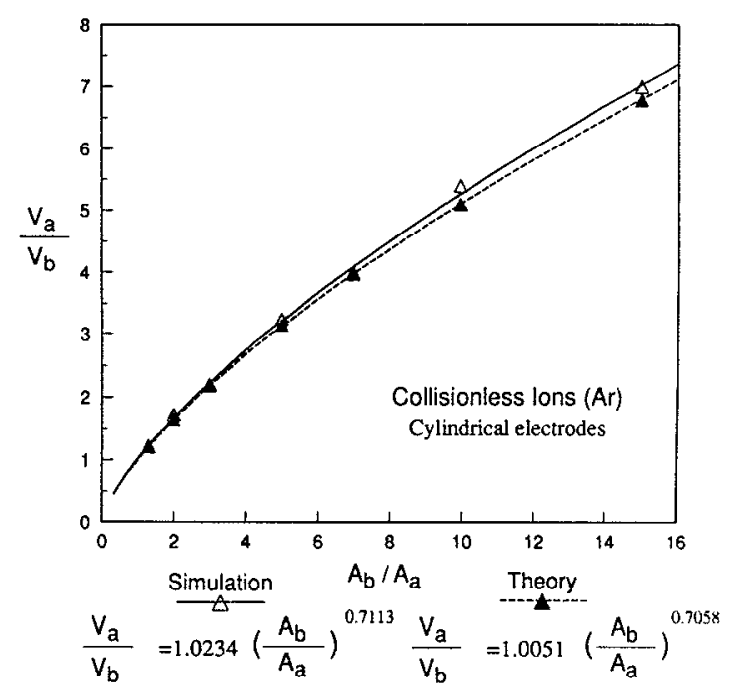

FIG. 7. $V_{a} / V_{b}$ vs $A_{b} / A_{a}$ for argon gas at $30 \mathrm{mT}$ Torr (collisionless ions in the simulation and the theory assumes a collisionless sheath model). 


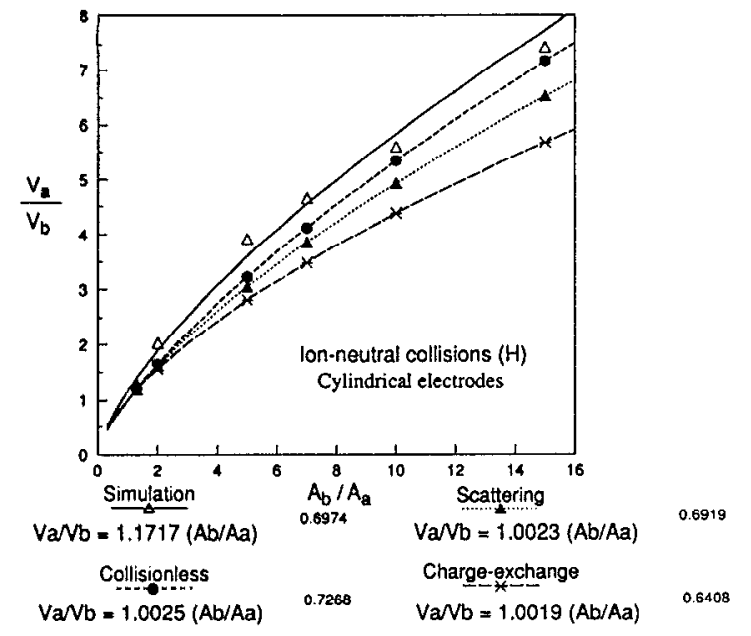

FIG. 8. $V_{a} / V_{b}$ vs $A_{b} / A_{a}$ for hydrogen gas at $30 \mathrm{mTorr}$ (ion-neutral collisions in the simulation). The theoretical points were obtained assuming the different models for the sheath, as indicated.

solving Eqs. (9), (10), and (12) numerically, assuming a collisionless sheath and using the electron temperature and peak-to-peak potential obtained by the simulations. We observe that the best fit for the points, shown in Figs. 6 and 7 , is somewhat different from Eq. (12) and the exponent in Table I. The discrepancy may be due to the inclusion of the floating potential [not included in Eq. (12)] in numerical calculations. We also observe that the difference introduced by the floating potential is more noticeable for argon then for hydrogen, since the electron temperature is higher for argon. Typically $T_{e} \approx 0.4-0.6 \mathrm{eV}$ in hydrogen and $T_{\mathrm{e}} \approx 2-3 \mathrm{eV}$ in argon.

Figure 8 shows the results for hydrogen, where ionneutral collisions were included in the simulation, for cylindrical electrodes. This figure suggests that the best agreement is given by the theoretical points obtained assuming a collisionless ion sheath model, even though the inner electrode sheath thicknesses measured in the simulations are several ion-neutral mean free path: $s_{a}$ $\approx 10-15 \lambda_{i}$. This may be the result of the assumption in our model, $V_{f a(b)}<V_{a}$, which is not always observed in the simulations.

The parameters for the simulation were an applied potential of $500 \mathrm{~V}$, if frequency of $1 \times 10^{7} \mathrm{H}$ and pressure of $30 \mathrm{mTorr}$. In the simulation, the pressure and the neutral temperature (in all runs considered as the room temperature) are used to determine the neutral density which is then used to determine the collision probability of a given particle. The neutral density is considered constant and uniform in space. The final electron temperature depends on the gas and also on the pressure used.

Note that the derivation of Eq. (12) does not consider any particular electrode shape, if we consider $s_{a}<r_{a}$. However, simulations using spherical electrodes done using the same general parameters (electrode separation, pressure and background gas) do not show the same agreement with theory for large area ratios as for cylindrical electrodes. We still observe a localized ionization close to the
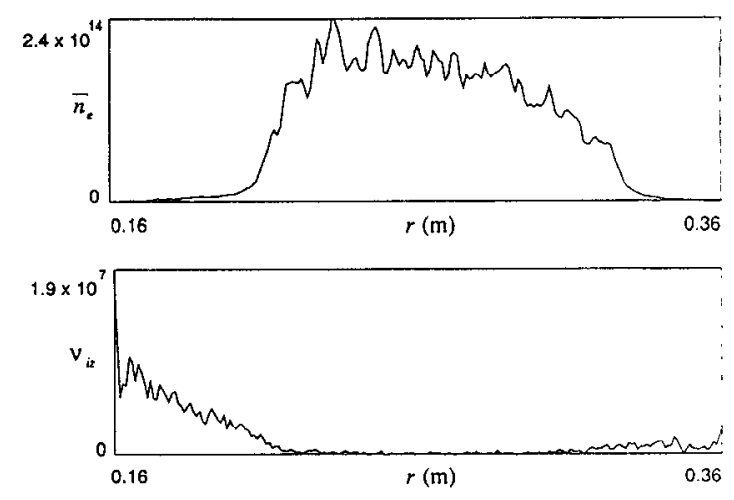

FIG. 9. (a) Typical spatial variation of the electron charge density averaged over an if period and (b) the ionization rate, $v_{i t}$, with spherical electrodes (cumulative in time), $A_{b} / A_{a}>3$. In this particular result the background neutral gas was hydrogen (collisionless ions) at $p=30$ mTorr and $A_{b} / A_{a}=5$.

powered electrode, as shown in Fig. 9, but the local ionization relationship (11), between densities at the sheaths and voltages, is no longer verified. Figure 10 shows $\left(n_{s a} V_{b}\right) /\left(n_{s b} V_{a}\right)$ versus area ratio for cylindrical and spherical electrodes. We observe that for cylindrical electrodes $\left(n_{s a} V_{b}\right) /\left(n_{s b} V_{a}\right)$ is very close to one, as expected from Eq. (11), up to an area ratio of 10. For spherical electrodes, the local ionization assumption breaks down for area ratios greater than about 3 .

The discrepancy in the scaling of $V_{a} / V_{b}$ with area ratio observed for spherical electrodes is due to the very small peak in the ionization rate seen at the outer electrode; i.e., the local ionization model is no longer valid, and the density $n_{s b}$ at the outer electrode may be determined by diffusion of plasma generated near the inner electrode, and not by ionization due to local stochastic heating at the outer electrode. For small area ratios, $A_{b} / A_{a}<3$, the relationship (11) is nearly verified and we observe reasonable compar-

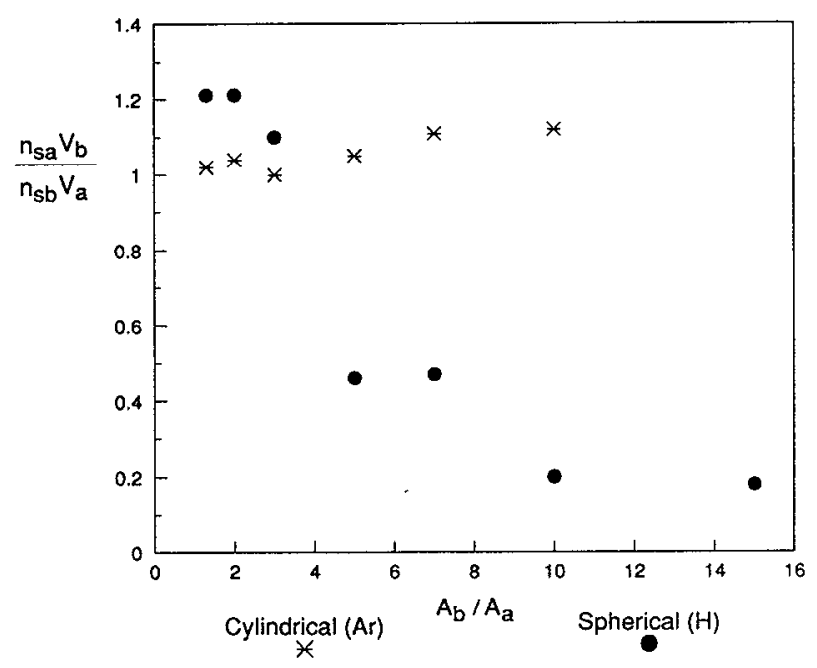

FIG. 10. $\left(n_{s b} V_{b}\right) /\left(n_{s b} V_{a}\right)$ vs $A_{b} / A_{a}$, for cylindrical and spherical electrodes. 


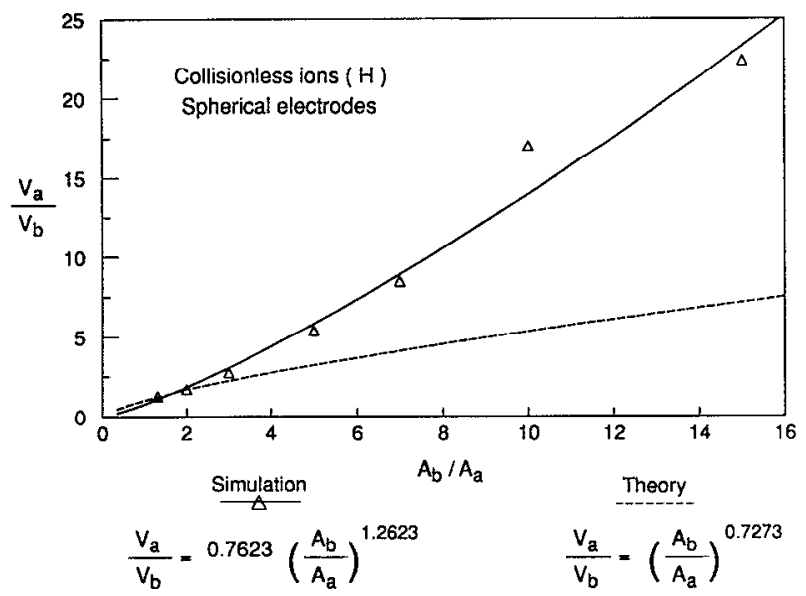

FIG. 11. $V_{a} / V_{b}$ vs $A_{b} / A_{a}$ for hydrogen gas (collisionless ions in the simulation), with spherical electrodes.

ison between the simulation and the theoretical results obtained assuming a collisionless sheath, in Figs. 11 and 12.

\section{SIMULATION RESULTS FOR BIAS VOLTAGE RATIOS}

The quantity measured in most experiments is the "bias ratio," defined as the bias voltage $V_{\text {bias }}=-\left(V_{a}\right.$ $\left.+V_{b}\right)$, normalized by the peak-to-peak rf voltage, $V_{p p}$. Thus, we present simulation results for this ratio to be used for comparison with experiments. Figures 13-15 show the simulation results for bias ratio versus inverse area ratio for all the runs. Although we have conditions differing from experimental bias measurement results (the electrode shapes are different and the final electron temperature in the simulation is lower then in the experiments, which results in a negative floating potential), we observe that our results are in the same range of magnitude as typical experimental results (e.g., see Ref. 11).

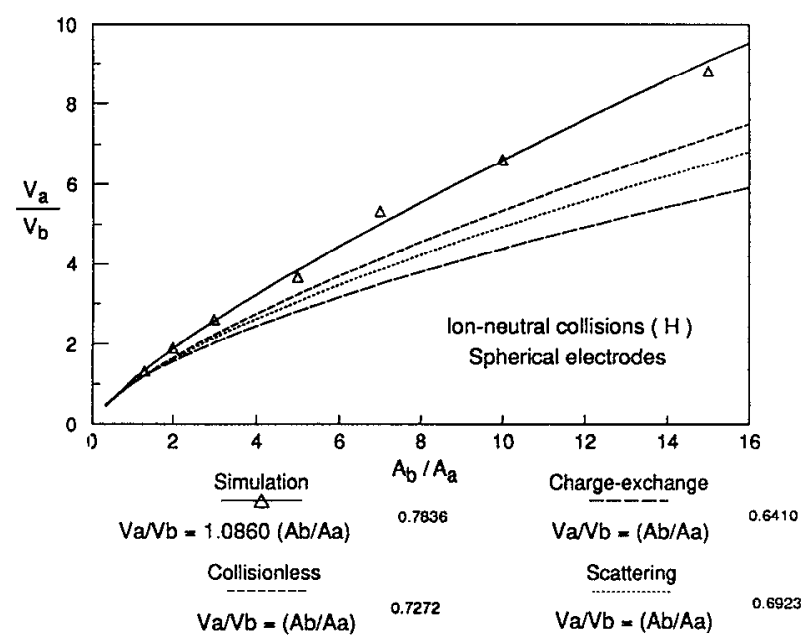

FIG. 12. $V_{a} / V_{b}$ vs $A_{b} / A_{a}$ for hydrogen gas (ion-meutral collisions in the simulation), with spherical electrodes.

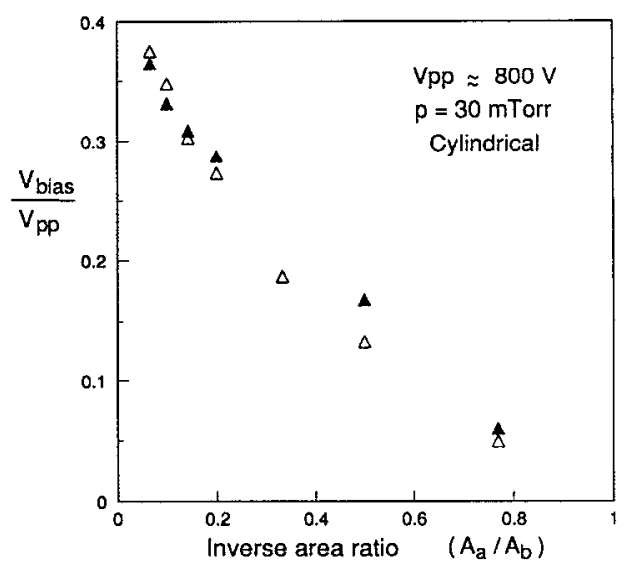

$\begin{array}{ll}\text { (H) Collisionless ions } & (H) \text { lon-neutral collisions }\end{array}$

FIG. 13. $V_{\text {bias }}$ vs $A_{a} / A_{b}$ for hydrogen gas (collisionless ions and ionneutral conditions in the simulation), with cylindrical electrodes.

\section{CONCLUSIONS}

The relationship between the voltage ratio and area ratio in asymmetric if discharges has been studied using spherical and cylindrical many-particle models and two different background gases at a single pressure of 30 $\mathrm{m}$ Torr. Over a limited range of area ratios it was found that the power dependence was close to one, not four, as a collisionless uniform ionization discharge model predicts. A local ionization model is in good agreement with the simulations for those area ratios where local ionization was observed in the simulations. The transition to a nonlocal model must be studied analytically in order to compare the simulations with an analytical model in this regime. We observe that the simulation results agree very well with theory for small area ratios, $A_{b} / A_{a}<3$, independent of the geometry used. The model used for the sheath in this range does not play a very important role (see Figs. 9 and 13).

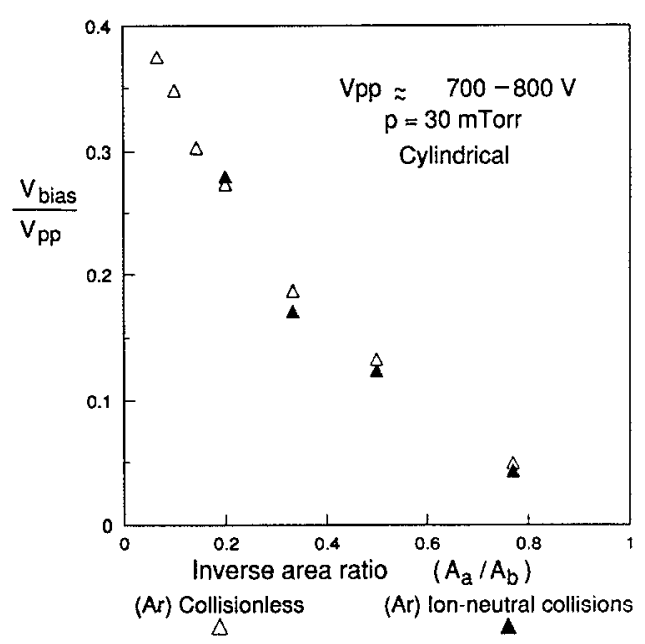

FIG. 14. $V_{\text {bias }}$ vs $A_{a} / A_{b}$ for argon gas (collisionless ions and ion-neutral collisions in the simulation), with cylindrical electrodes. 


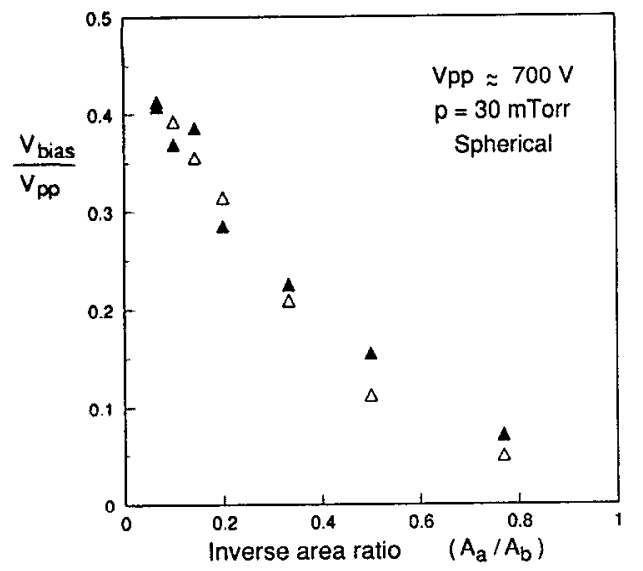

(H) Collisionless ions

(H) lon-neutral collisions

FIG. 15. $V_{\text {bias }}$ vs $A_{a} / A_{b}$ for hydrogen gas (collisionless ions and ionneutral collisions in the simulation), with cylindrical electrodes.

Additional simulations at pressures both above and below $30 \mathrm{mT}$ Torr are needed in order to test the validity of the analytical model.

\section{ACKNOWLEDGMENTS}

This work is supported in part by ONR Contract No. N00014-90-J-1198, NSF Grant ECS-8517363 and DOE

Grant DE-FG03-87ER13727. M. V. Alves supported partially by CAPES-Ministry of Education, Brazil.

${ }^{1}$ B. Chapman, Glow Discharge Processes-Sputtering and Plasma Etching (Wiley, New York, 1980).

${ }^{2}$ C. M. Horwitz, J. Vac. Sci. Technol. A 1, 60 (1983); K. Kohler, J. W. Coburn, D. E. Horne, E. Kay, and J. H. Keller, J. Appl. Phys. 57, 59 (1985).

${ }^{3}$ Codes available from Industrial Liaison Program, EECS Dept., UC Berkeley.

${ }^{4}$ M. V. Alves, Study of Asymmetric Electrical Discharges Using Particle Simulations, INPE, Brazil, Sept. 1990 (Ph.D thesis in Portuguese).

${ }^{5}$ C. K. Birdsall and A. B. Langdon, Plasma Physics Via Computer Simulation (McGraw-Hill, New York, 1985).

${ }^{6}$ W. S. Lawson, J. Comp. Phys. 80, 253 (1989).

${ }^{7}$ J. P. Verboncoeur, M. V. Alves, and V. Vahedi, J. Comp. Phys. (1990). ${ }^{8}$ W. H. Press, B. P. Flannery, S. A. Teukolsky, and W. T. Vetterling, Numerical Recipes in C-The Art of Scientific Computing (Cambridge University Press, London, 1988)

${ }^{9}$ A. M. Pointu, Appl. Phys. Lett. 50, 1047 (1987).

${ }^{10}$ M. A. Lieberman, J. Appl. Phys. 65, 4186 (1989).

${ }^{11}$ M. A. Lieberman and S. E. Savas, J. Vac. Sci. Technol. A 8, 1632 (1990).

${ }^{12}$ V. A. Godyak, Soviet Radio Frequency Discharge Research (Delphic, Falls Church, VA, 1986), p. 86.

${ }^{13}$ J. H. Keller and W. B. Pennebaker, IBM J. Res. Dev, 23, 3 (1979).

${ }^{14}$ A. Garscadden and K. G. Emeleus, Proc. Phys. Soc. 79, 535 (1962).

${ }^{15} \mathrm{~F}$. F. Chen, Introduction to Plasma Physics and Controlled Fusion (Plenum, New York, 1984), p. 292.

${ }^{16}$ J. D. Cobine, Gaseous Conductors (Dover, New York, 1941), p. 128. 